\title{
Structural Change and Generalized Balanced Growth
}

\author{
Jürgen Meckl* \\ University of Konstanz
}

November 1999

\begin{abstract}
This paper addresses the criticism that balanced growth models are inconsistent with the dynamics of structural change typical for the process of economic growth. Using a sectoral disaggregated version of a researchdriven growth model, we develop the concept of a generalized balanced growth path (GBGP). The GBGP preserves decisive properties of the balanced growth path analyzed in aggregated growth models. Along a GBGP, aggregate variables grow at constant rates (balanced growth) while disaggregated variables grow at non-constant rates. The aggregated model's implications for the behavior of aggregates are robust with respect to structural dynamics.
\end{abstract}

JEL Classification O14, O41

Keywords Structural adjustment, innovation-based growth

\footnotetext{
*Correspondence: Jürgen Meckl, University of Konstanz, Faculty of Economics and Statistics, Fach D 146, D-78457 Konstanz, Germany, Phone: +49-(0)7531-88-2918, Fax: -4558, email: juergen.meckl@uni-konstanz.de. For useful comments, I am grateful to Max Albert, Bodo Hilgers, Hans Jürgen Ramser, Norbert Wunner, and Stefan Zink.
} 


\section{Introduction}

The actual development of real gross domestic product (GDP) per capita observed in several industrialized countries (especially in the U.S.) over the last 125 years can be remarkably well approximated by an exponential growth path (cf. Jones 1997). Thus it comes at no surprise that proponents of growth theory consider a balanced growth path (BGP) as an adequate description of the process of economic growth. Additional support for this view of the economy as being on or at least very close to a BGP comes from a number of stylized facts characterizing economic development such as the absence of trends in the real interest rate (cf. Kaldor 1963). That kind of empirical evidence is put forward as a justification for theoretical work concentrating on the analysis of a BGP. This is especially true for a number of models of endogenous growth that completely abstract from any form of transitional dynamics, as, e.g., in the one-factor models of Grossman \& Helpman (1991), Rivera-Batiz \& Romer (1991), Aghion \& Howitt (1992), or the one-factor version of the Romer (1990) model discussed by Barro \& Sala-iMartin (1995). By abstracting from any form of investment in the accumulation of production factors, these models transform the essentially dynamic problem describing economic development into a static problem, thereby simplifying the technical analysis considerably. ${ }^{1}$

The concentration on the analysis of a BGP, though convenient from an analytical point of view, has been criticized on the grounds that it rules out important phenomena by mere assertion (cf. Aghion \& Howitt 1998: ch. 2). Along a BGP, all variables grow at constant rates, and the economy of tomorrow will always be a scaled-up version of today's economy. But this implication of balanced growth is clearly at odds with empirical observations. Several authors (cf. Clark 1957, Chenery 1960, Kuznets 1971, Baumol et al. 1989, or Kongsamut et al. 1997) report on the dramatic change in sectoral composition of output that occurred over the last century in most industrialized countries. These facts about structural change are a serious challenge for the traditional view of balanced growth, and hence for predictions derived from models concentrating on the analysis of BGP.

Echevarria (1997) and Kongsamut et al. (1997) argue for a sectorally disag-

\footnotetext{
${ }^{1}$ This also applies to several analyses based on two-factor versions of the model developed by Romer (1990). Although this model allows for accumulation of factors by investment in capital, the complex transitional dynamics are typically ignored.
} 
gregated growth model which allows for structural change along the growth path. Specifically, both papers develop a sectoral disaggregated version of the neoclassical growth model and analyze structural change originating in nonhomothetic consumer preferences. ${ }^{2}$ These models, however, are hard to reconcile with the observation that even massive changes in sectoral composition of an economy seem to have only minor effects on the long-run aggregate development of economies (especially in the U.S.), as indicated by the data. Although in both models the economy converges towards a BGP, structural adjustment almost comes to a halt as the economy approaches the BGP. As a result, these models are incompatible with the stylized facts of the growth process, namely the simultaneous existence of exponential growth in aggregates and nonnegligible structural change.

The present paper analyzes structural adjustment using a R\&D-driven growth model similar to Romer (1990) and Grossman \& Helpman (1991). We allow for structural adjustment in the process of economic growth by disaggregating the final-goods sector. As in the work of Echevarria and Kongsamut et al., structural change originates in nonhomothetic preferences. In contrast to their work, however, our model delivers a generalized balanced growth path (GBGP) that can explain the simultaneous existence of balanced growth in aggregate variables and change in the sectoral composition of the economy. Specifically, we require that along a GBGP (i) all relative goods' prices and factor prices are constant, and (ii) the share of ressources allocated to research and manufacturing (the production of final goods and of intermediate goods) remains fixed over time. ${ }^{3}$ This generalization of the BGP concept allows for structural change since the allocation of ressources within the sector producing final goods is not required to be fixed. We show that with R\&D taking the form of raising total factor productivity, there exists a GBGP along which all aggregate variables grow at constant rates, whereas production and, of course, consumption of each final good grow at different rates.

\footnotetext{
${ }^{2}$ These two models differ only with respect to the specification of the utility function. Due to the complexity of the utility function applied by Echevarria (1997), her model can be solved only numerically. Kongsamut et al. (1997) overcome this problem by specifying a less complex utility function which allows for an analytical analysis of growth paths.

${ }^{3}$ Note that our definition of a GBGP differs from the definition used by Kongsamut et al. The latter define a GBGP as a path where the real interest rate does not change over time. This definition is motivated by the Kaldor facts about economic growth. Our definition is more restrictive, but also more in the spirit of the ordinary definition of an equilibrium as a situation where relative prices do not change.
} 
Balanced growth in aggregate variables is consistent with structural adjustment in disaggregate variables.

By explicitly aggregating over variables we also show that the behavior of aggregates is correctly described by an appropriately specified non-disaggregated endogenous growth model. The aggregated model's implications for the behavior of aggregates are robust with respect to structural dynamics. Thus, our analysis refutes the conjecture by Kongsamut et al. (1997) that balanced-growth models are inconsistent with structural change. On the contrary, it is possible to reinterpret a standard growth model formulated in aggregate variables as a disaggregated model fully compatible with structural change while preserving the balanced-growth properties in aggregates. Our analysis indicates that as long as we are only interested in the behavior of aggregate variables, there is simply no need to disaggregate.

The paper is organized as follows. Section 2 presents our model and analyzes the instantaneous equilibrium. Section 3 introduces our concept of generalized balanced growth and works out the necessary conditions for existence of a GBGP with structural adjustment. In section 4 we show that a standard (BGP-) growth model follows from explicit aggregation of our disaggregated model with structural adjustment. Section 5 provides some concluding remarks.

\section{The model}

We model R\&D-driven economic growth using an idea-based growth model going back to the work of Romer (1990) and Grossman \& Helpman (1991). While for the present purpose it makes no difference whether technological change takes the form of expanding the number of products or of increasing the quality of a given number of products, we apply the product-variety approach. In order to account for structural adjustment, we disaggregate the production sector manufacturing final goods. These goods are assumed to be exclusively used for consumption purposes. Structural adjustment then is the change in the relative size of subsectors producing different final goods occurring simultaneously with a corresponding change in their shares in total consumption expenditure. As in the model of Echevarria (1997) and Kongsamut et al. (1997), structural adjustment is driven by differences in income elasticities of demand originating in nonhomoth- 
etic preferences. The production structure adjusts accordingly during the growth process.

\subsection{Consumption}

Consumption decisions are modelled by assuming a representative agent maximizing the utility function

$$
U(t)=\int_{t}^{\infty} \frac{C(\tau)^{1-\theta}-1}{1-\theta} e^{-\rho(\tau-t)} d \tau, \quad \theta>0,
$$

where $C$ denotes an aggregator function for the consumption of final goods, $\rho$ the rate of time preference, and $\theta$ stands for the inverse of the elasticity of substitution. In specifying the consumption-aggregator function we follow Kongsamut et al. (1997) and assume $C$ to be of the form

$$
\ln C=\sum_{i=1}^{N} \beta_{i} \ln \left(C_{i}+\bar{C}_{i}\right), \quad \sum_{i=1}^{N} \beta_{i}=1 .
$$

Here, $C_{i}$ denotes the consumption of final good $i$ and the $\bar{C}_{i}$ are parameters. This specification covers the homothetic Cobb-Douglas form as a special case $\left(\bar{C}_{i}=0\right.$ for all $i=1, \ldots, N)$ with an income elasticity of demand equal to unity for all goods. In general, income elasticities exceed (are less than) unity for goods with $\bar{C}_{i}>0\left(\bar{C}_{i}<0\right)$.

The vector of factor supplies $\boldsymbol{L}=\left(L_{1}, \ldots, L_{M}\right)$ is assumed to be given exogenously. The representative agent chooses time paths of $C_{i}$ that maximize (1) while respecting the dynamic budget constraint

$$
\dot{W}=r W+\boldsymbol{w} \cdot \boldsymbol{L}-\boldsymbol{p} \cdot \boldsymbol{C},
$$

where $W$ is the value of the household's asset holdings, and $r$ is the interest rate. Aggregate factor income is given by the vector product $\boldsymbol{w} \cdot \boldsymbol{L}$, where $\boldsymbol{w}$ is the vector of factor prices $\left(w_{1}, \ldots, w_{M}\right)$. Consumption expenditure is given by $E \equiv \boldsymbol{p} . \boldsymbol{C}$, where $\boldsymbol{p}$ is the vector of final-goods' prices $\left(p_{1}, \ldots, p_{N}\right)$, and $\boldsymbol{C}$ is the vector of final-goods' demand $\left(C_{1}, \ldots, C_{N}\right)$.

Denoting the shadow price of the dynamic constraint by $\lambda$, the FOCs of the consumer problem are

$$
\begin{aligned}
\beta_{i} \frac{C^{1-\theta}}{C_{i}+\bar{C}_{i}} & =\lambda p_{i}, \quad i=1, \ldots, N \\
\dot{\lambda} & =\lambda(\rho-r)
\end{aligned}
$$


(3) determines the optimal allocation of consumer goods as

$$
C_{j}+\bar{C}_{j}=\frac{\beta_{j} p_{i}}{\beta_{i} p_{j}}\left(C_{i}+\bar{C}_{i}\right) \quad \forall i, j=1, \ldots, N
$$

(5) defines the expansion path of consumption for a given vector of goods' prices $\boldsymbol{p}$. Due to the specification of $C$, there is a linear relation between each two pairs of consumer demands. Considering only two consumer goods $i=1,2$, the expansion path is a straight line with a slope $-\beta_{2} p_{1} /\left(\beta_{1} p_{2}\right)$ in $\left(C_{1}, C_{2}\right)$ space. Only in the case of an homothetic aggregator function $C$, this line passes through the origin. In general, (5) defines a family of parallel lines. This means that the values $\bar{C}_{i}$ in the consumption index just shift the origin of the system of coordinates in a certain direction. As a consequence of this shift, relative consumption expenditures $p_{i} C_{i} / p_{j} C_{j}$ converge towards the fixed value $\beta_{i} / \beta_{j}$ as income grows over time. This implies that - given the price vector $\boldsymbol{p}$ - the growth rates of demand for the different consumption goods converge over time.

We can use the FOCs to determine the growth rate of $C$ and the growth rate of consumption expenditure $E$. Substituting in (2) for $C_{j}$ according to (5) gives

$$
\ln C=\ln \left(C_{i}+\bar{C}_{i}\right)-\ln \frac{\beta_{i}}{p_{i}}+\sum_{j=1}^{n} \beta_{j} \ln \frac{\beta_{j}}{p_{j}} .
$$

Given a price vector $\boldsymbol{p}$, the growth rates of the $C_{i}$ and the growth rate of $C$ are related by

$$
\frac{\dot{C}_{i}}{C_{i}}=\frac{C_{i}+\bar{C}_{i}}{C_{i}} \frac{\dot{C}}{C}
$$

In general, the growth rates for the various $C_{i}$ differ in the case of a nonhomothetic consumption aggregator function. Consumption of final goods with an income elasticity of demand exceeding unity grows at a rate exceeding the growth rate of the consumption aggregator and vice versa. These differences in growth rates, however, diminish over time. As a result, the demand of goods with high income elasticities rises relative to the demand of goods with low income elasticities, but converges to a fixed value.

In order to relate the growth rate of $C$ to the interest rate, we differentiate (3) with respect to time:

$$
(1-\theta) \frac{\dot{C}}{C}-\frac{C_{i}}{C_{i}+\bar{C}_{i}} \frac{\dot{C}_{i}}{C_{i}}=\frac{\dot{\lambda}}{\lambda}
$$


From (4), (6) and (7) we find that the following condition holds for the aggregator function for consumption:

$$
g_{C} \equiv \frac{\dot{C}}{C}=\frac{r-\rho}{\theta} .
$$

This is exactly the same condition that determines the time path of consumption in a model with a single consumer good. In the present model, however, $C$ is not equal to consumption expenditure. Note that our specification of the aggregator function for consumption ensures that differences in growth rates of demand for each consumer good do not affect the growth rate of the consumption aggregator.

Whether a situation with constant growth rates in aggregate variables is compatible with nonconstant growth rates in disaggregated variables will turn out to depend on whether the aggregator function for consumption $C$ and aggregate consumption expenditure $E$ grow at the same rate. Given the price vector $\boldsymbol{p}, E$ grows at rate

$$
g_{E} \equiv \frac{\dot{E}}{E}=\sum_{i} \frac{p_{i} C_{i}}{E} \frac{\dot{C}_{i}}{C_{i}} .
$$

Substituting for $\dot{C}_{i} / C_{i}$ according to (6) yields

$$
g_{E}=\sum_{i} \frac{p_{i}\left(C_{i}+\bar{C}_{i}\right)}{E} g_{C} .
$$

Thus, $E$ and $C$ grow at the same rate iff $\sum p_{i} \bar{C}_{i}=0$. Obviously, the special case of a homothetic aggregator function for consumption always satisfies this condition.

\subsection{Production}

The production side of the economy consists of three sectors: a final-goods sector, an intermediate-goods sector, and a research sector. The final-goods sector is made up by a number of $N$ subsectors producing goods that are used solely for consumption purposes (in the case of $N=3$, we may interpret the sectors as agriculture, manufacturing, and services). In each final-goods subsector $i$, perfectly competitive firms produce a homogeneous final good using primary factors and a number of different varieties of intermediate goods. At any instant, $A$ measures the range of intermediate goods that are available to producers of final goods. The output in each subsector, $Y_{i}$, is given by the production function

$$
Y_{i}=\left[F_{i}\left(\boldsymbol{L}_{i}\right)\right]^{1-\alpha} \int_{0}^{A}\left[x_{i}(\omega)\right]^{\alpha} d \omega, \quad 0<\alpha<1, i=1, \ldots, N .
$$


$F_{i}$ is a linearly homogeneous function of sector $i$ 's vector of factor inputs $\boldsymbol{L}_{i}=$ $\left(L_{1 i}, \ldots, L_{M i}\right)$, and $x_{i}(\omega)$ denotes the input of intermediate good $\omega$ in the finalgoods subsector $i$. In order to ensure that $R \& D$ can be interpreted as technological progress altering total factor productivity, the partial elasticities of production with respect to intermediate goods are assumed to be identical for all final-goods subsectors.

The unit-cost functions associated with these technologies are

$$
c_{i}\left(\boldsymbol{w}, p_{D}\right) \equiv\left[\tilde{c}_{i}(\boldsymbol{w})\right]^{1-\alpha} p_{D}^{\alpha}, \quad i=1, \ldots, N
$$

where $p_{D}$ is a price index for intermediates given by

$$
p_{D}=\left(\int_{0}^{A}[p(\omega)]^{\alpha /(\alpha-1)} d \omega\right)^{(\alpha-1) / \alpha} .
$$

Denoting the price of final good $i$ by $p_{i}$, the zero-profit conditions can be written as

$$
p_{i}=c_{i}\left(\boldsymbol{w}, p_{D}\right), \quad i=1, \ldots, N .
$$

From our assumption on technologies it follows that the unit-cost functions $\tilde{c}_{i}(\boldsymbol{w})$ and $c_{i}\left(\boldsymbol{w}, p_{D}\right)$ are linearly homogeneous functions. Applying the envelope theorem to the unit-cost function $c_{i}$ gives sector $i$ 's demand for intermediate goods as

$$
x_{i}(\omega)=\alpha p_{i} Y_{i}\left(\frac{p_{D}^{\alpha}}{p(\omega)}\right)^{1 /(1-\alpha)}, \quad i=1, \ldots, N
$$

Aggregating over all final-goods subsectors, the total demand for each variety $\omega$ of the intermediate good is

$$
x(\omega)=\alpha \boldsymbol{p} . \boldsymbol{Y}\left(\frac{p_{D}^{\alpha}}{p(\omega)}\right)^{1 /(1-\alpha)},
$$

where $\boldsymbol{p} . \boldsymbol{Y}$ is the vector product of the price vector $\boldsymbol{p}=\left(p_{1}, \ldots, p_{N}\right)$ and the final-goods output vector $\boldsymbol{Y}=\left(Y_{1}, \ldots, Y_{N}\right)$.

Each variety of the intermediate goods is produced by a single firm owning a perpetual monopoly right (an infinitely lived patent) for the use of a certain previously invented design necessary for production. Designs are either developed in a firm's own R\&D department or they are purchased from firms specializing in R\&D. Once designs are developed or purchased, producers combine primary 
factors to produce intermediate goods. We assume identical constant-returnsto-scale technologies for all intermediate producers. Each intermediate producer chooses the price of his product to maximize profits, taking $\boldsymbol{p} . \boldsymbol{Y}$ and $p_{D}$ as given (monopolistic competition). The problem of the producer of intermediate good $\omega$ then is

$$
\max _{p(\omega)}\left[p(\omega)-c_{x}(\boldsymbol{w})\right] \alpha \boldsymbol{p} . \boldsymbol{Y}\left(\frac{p_{D}^{\alpha}}{p(\omega)}\right)^{1 /(1-\alpha)},
$$

where $c_{x}$ is the unit-cost function for producing intermediates. The solution of this problem yields an equilibrium price for each intermediate of

$$
p(\omega)=c_{x}(\boldsymbol{w}) / \alpha \equiv p_{x}
$$

With all intermediates bearing the same price, they are all produced in equal quantities, and $p_{D}$ is given by

$$
p_{D}=p_{x} A^{(\alpha-1) / \alpha}=\frac{c_{x}(\boldsymbol{w})}{\alpha} A^{(\alpha-1) / \alpha} .
$$

With (16) and (17), the profits of an intermediate producer, $\pi_{x}$, turn out to be a linear function of $\boldsymbol{p .} \boldsymbol{Y} / A$ :

$$
\pi_{x}=\alpha(1-\alpha) \boldsymbol{p} . \boldsymbol{Y} / A
$$

Due to patent protection, these profits cannot be driven to zero by imitation. Producers of intermediates realize a stream of future profits with a present value of

$$
V(t)=\int_{t}^{\infty} \pi_{x}(\tau) e^{-\int_{t}^{\tau} r(s) d s} d \tau .
$$

Differentiating (19) with respect to time gives

$$
\dot{V}=r V-\pi \text {. }
$$

This condition equating the expected capital gains and dividend payments (firm profits) received by shareholders of a representative $R \& D$ firm to the yields on a riskless loan describes the capital-market equilibrium.

Profits of firms producing intermediates are extracted by the cost of developing new designs or purchasing these from firms specializing in R\&D. Firms in the research sector use only primary factors to invent designs for new intermediate goods according to the production function

$$
g_{A} \equiv \frac{\dot{A}}{A}=F_{R}\left(\boldsymbol{L}_{R}\right) \text {. }
$$


Here, $g_{A}$ denotes the growth rate of the number of varieties of intermediates, the rate of innovation. $F_{R}$ is a linearly homogeneous function of the vector of factor inputs $\boldsymbol{L}_{R}=\left(L_{1 R}, \ldots, L_{M R}\right)$ employed in the research sector. As in the models of Romer (1990) and Grossman \& Helpman (1991), long-run growth originates in the fact that the research technology is linearly homogeneous in the public good "knowledge" (the number of intermediate goods that have been already invented). The only difference to their work is that we allow for $M$ factors to be used in R\&D.

We assume free entry into the R\&D sector. Firms in the R\&D sector finance the upfront costs of developing new designs by issuing shares on the equity market. Since the shares issued by R\&D firms are the only asset in the economy, we have $W=A V$. Successful inventors either become producers of intermediates or charge a royalty for the use of the design. In any case, the $R \& D$ costs are covered by the present value of future profits that accrue to a producer of intermediates. Denoting the unit-cost function associated with the R\&D technology $F_{R}\left(\boldsymbol{L}_{R}\right)$ by $c_{R}(\boldsymbol{w})$, free entry into the research sector implies that

$$
\frac{c_{R}(\boldsymbol{w}(t))}{A(t)} \geq V(t)
$$

with equality in equilibria with positive $R \& D$. In the following, we concentrate on equilibria with positive $\mathrm{R} \& \mathrm{D}$ and, hence, growing $A$ at all points in time.

\subsection{Equilibrium}

The model is closed by the equilibrium conditions for factor markets and consumer goods markets. Before analyzing these market-clearing conditions in detail, let us have a closer look at firms' unit-cost functions first. From the homogeneity properties of the unit-cost functions it follows that

$$
\begin{aligned}
\tilde{c}_{i}(\boldsymbol{w}) & =\tilde{c}_{i}\left(A^{\alpha-1} \boldsymbol{w}\right) A^{1-\alpha} \equiv \tilde{c}_{i}(\overline{\mathbf{w}}) A^{1-\alpha} \\
c_{x}(\boldsymbol{w}) & =c_{x}\left(A^{\alpha-1} \boldsymbol{w}\right) A^{1-\alpha} \equiv c_{x}(\overline{\mathbf{w}}) A^{1-\alpha} \\
c_{R}(\boldsymbol{w}) & =c_{R}\left(A^{\alpha-1} \boldsymbol{w}\right) A^{1-\alpha} \equiv c_{R}(\overline{\mathbf{w}}) A^{1-\alpha}
\end{aligned}
$$

where $\overline{\mathbf{w}} \equiv A^{\alpha-1} \boldsymbol{w}$ denotes the vector of productivity-adjusted factor prices. Substituting for $p_{D}$ in the unit-cost function of final-goods producers according 
to (17) implies

$$
c_{i}\left(\boldsymbol{w}, p_{D}\right)=\left[\tilde{c}_{i}(\boldsymbol{w})\right]^{1-\alpha}\left[\frac{c_{x}(\boldsymbol{w})}{\alpha}\right]^{\alpha} A^{\alpha-1} .
$$

Substituting for $\tilde{c}_{i}(\boldsymbol{w})$ and $c_{x}(\boldsymbol{w})$ according to (23) and (24) gives

$$
\begin{aligned}
c_{i}\left(\boldsymbol{w}, p_{D}\right) & =\left[\tilde{c}_{i}(\overline{\mathbf{w}}) A^{1-\alpha}\right]^{1-\alpha}\left[\frac{c_{x}(\overline{\mathbf{w}}) A^{1-\alpha}}{\alpha}\right]^{\alpha} A^{\alpha-1} \\
& =\left[\tilde{c}_{i}(\overline{\mathbf{w}})\right]^{1-\alpha}\left[\frac{c_{x}(\overline{\mathbf{w}})}{\alpha}\right]^{\alpha} \equiv c_{i}(\overline{\mathbf{w}}) .
\end{aligned}
$$

Due to our assumption of identical values $\alpha$ for all sectors producing final goods, the reduced form of the unit-cost function $c_{i}$ is only a function of productivityadjusted factor prices. The impact of the state of technology (as measured by $A$ ) is only indirect insofar as it affects factor prices. For this reason we can refer to innovation of new intermediates as neutral technological progress raising total factor productivity in all final-goods sectors equally. Finally, substituting for $c_{i}\left(\boldsymbol{w}, p_{D}\right)$ from $(26)$ in (13) gives the zero-profit conditions as

$$
p_{i}=c_{i}(\overline{\mathbf{w}}), \quad i=1, \ldots, N
$$

Applying the envelope theorem to the reduced form of the unit-cost function $c_{i}$ allows us to derive the factor-input coefficients as

$$
\frac{\partial c_{i}(\overline{\mathbf{w}})}{\partial w_{l}}=b_{l i}(\overline{\mathbf{w}}) A^{\alpha-1} \text {. }
$$

The input coefficients $b_{l i}$ measure the direct and indirect (the factor input embodied in intermediates) input of primary factor $l$ in final-goods sector $i$. These coefficients depend only on relative factor prices. The same applies for the factorinput coefficients in the R\&D sector $a_{l R}(\overline{\mathbf{w}})$ which can be derived by applying the envelope theorem to the unit-cost function $c_{R}(\overline{\mathbf{w}})$. Factor inputs of the R\&D sector are then given by $\dot{A} a_{l R}(\overline{\mathbf{w}}) / A$.

With an exogenously given vector of factor supplies $\boldsymbol{L}$, the conditions for factor-market clearing can then be written as

$$
g_{A} \boldsymbol{a}+\boldsymbol{B} \overline{\mathbf{Y}}=\boldsymbol{L}
$$

where $\boldsymbol{a}$ is the $(M \times 1)$-vector of input coefficients $a_{l R}(\overline{\mathbf{w}}), \boldsymbol{B}$ is the $(M \times N)$ matrix of input coefficients $b_{l i}(\overline{\mathbf{w}}), \overline{\mathbf{Y}}$ is the vector of final-good outputs adjusted for changes in total factor productivity, and $\bar{Y}_{i} \equiv Y_{i} A^{\alpha-1}$. 
It turns out to be useful to define a maximum-value function $\phi\left(\boldsymbol{p}, \boldsymbol{L}, g_{A}\right)$ that summarizes the production-sector equilibrium, i.e. the zero-profit conditions for producers of final goods and the factor-market-clearing conditions. We define this function by the optimization problem

$$
\phi\left(\boldsymbol{p}, \boldsymbol{L}, g_{A}\right) \equiv \min _{\overline{\mathbf{w}}}\left\{\overline{\mathbf{w}} \cdot \boldsymbol{L}-g_{A} c_{R}(\overline{\mathbf{w}}): c_{i}(\overline{\mathbf{w}})=p_{i}\right\}
$$

Interpreting the Lagrange multipliers of the constraints as the productivityadjusted outputs of final goods, the FOCs of this problem are given by (27) and (29). The following properties of the function $\phi\left(\boldsymbol{p}, \boldsymbol{L}, g_{A}\right)$ will turn out to be useful in the analysis of the intertemporal allocation. It is non-decreasing in factor endowments $\boldsymbol{L}$, it is non-increasing in the growth rate $g_{A}$, and it is concave and linearly homogeneous in $\left(\boldsymbol{L}, g_{A}\right)$. The latter properties follow from the fact that the objective function is linear in $\boldsymbol{L}$ and in $g_{A}$, while $\boldsymbol{L}$ and $g_{A}$ do not enter the constraints.

The value of $\phi\left(\boldsymbol{p}, \boldsymbol{L}, g_{A}\right)$ is the difference of the productivity-adjusted values of aggregate factor income and total $\mathrm{R} \& \mathrm{D}$ costs. The following argument shows that this value is proportional to the aggregate revenue in the final-goods sector. Due to the homogeneity properties of all technologies, the aggregate revenue in the final goods sector is exhausted by aggregate factor payments in the finalgoods sector and in the intermediate-goods sector plus the aggregate profits in the intermediate-goods sector. Making use of (18), this condition can be written as

$$
\begin{gathered}
\boldsymbol{p} . \boldsymbol{Y}=\boldsymbol{w} . \boldsymbol{L}-g_{A} c_{R}(\boldsymbol{w})+\alpha(1-\alpha) \boldsymbol{p} . \boldsymbol{Y} \\
\Longleftrightarrow \\
\boldsymbol{p} . \overline{\mathbf{Y}}=\gamma\left[\overline{\mathbf{w}} \cdot \boldsymbol{L}-g_{A} c_{R}(\overline{\mathbf{w}})\right],
\end{gathered}
$$

where $\gamma \equiv[1-\alpha(1-\alpha)]^{-1}>0$. Thus, we can define the net GDP function $\bar{y}\left(\boldsymbol{p}, \boldsymbol{L}, g_{A}\right)$ by

$$
\bar{y}\left(\boldsymbol{p}, \boldsymbol{L}, g_{A}\right) \equiv \gamma \phi\left(\boldsymbol{p}, \boldsymbol{L}, g_{A}\right) .
$$

Obviously, $\bar{y}$ equals the value of productivity-adjusted outputs $\boldsymbol{p} . \overline{\mathbf{Y}}$. The properties of the function $\phi($.$) carry over to the function \bar{y}($.$) .$

The equilibrium conditions are completed by the market-clearing conditions for final goods:

$$
Y_{i}=C_{i}, \quad i=1, \ldots, N
$$


This completes our specification of the model. At every moment in time, the number of existing intermediate goods, $A(t)$, is determined by history. Expectations about future profitability determine the value of the representative firm producing intermediates, $V(t)$. Agents take this value as exogenous. Given $A(t)$ and $V(t)$, the static equilibrium is described by the equilibrium of a standard Arrow-Debreu model. Since the preferences of the representative consumer are strictly convex and the production-possibility set is convex, there exists a unique solution for the equilibrium price vector. Provided that the economy is of sufficient size, this solution is characterized by full diversification, i.e. the output of all final-goods subsectors is positive.

\section{Generalized balanced growth}

In this section we demonstrate that the disaggregated version of a standard reseach-driven growth model developed in section 2 can explain structural adjustment along a growth path that is characterized by balanced-growth behavior in aggregate variables. We term this path that is consistent with these stylized facts of the growth process, a generalized balanced growth path (GBGP). In particular, we work out restrictions on consumer preferences and on the structure of the final-good sector which are necessary for a GBGP to exist.

\subsection{GBGP and equilibrium rate of innovation}

We define a GBGP as a growth path where both (i) all relative prices of goods (if required, as for intermediate goods, adjusted for changes in total factor productivity) and of factors and (ii) the shares of ressources allocated to research, production of intermediates and production of final goods remain fixed over time. Due to this definition, the growth rates of aggregate variables (per capita GDP, consumption expenditure, rate of innovation) will be constant along a GBGP. However, factor reallocation within the sector producing intermediate goods or within the final-goods subsectors is allowed on a GBGP. In contrast to the conventional definition of a BGP, which requires constant growth rates for all variables, growth along a GBGP is consistent with structural adjustment.

We assume that there are at least as many final goods as there are factors, i.e. $N \geq M$. In this case, any $M$ of the zero-profit conditions (27) determine 
the productivity-adjusted factor prices $\overline{\mathbf{w}}$ as a function of the final-good prices $p_{1}, \ldots, p_{M}$. The requirement that relative prices for final goods are constant along a GBGP implies constant prices $p_{i}$ for all $i=1, \ldots, N$. It follows that the productivity-adjusted factor prices are also constant implying that relative factor prices remain fixed along the GBGP. Real factor prices grow at rate $(1-\alpha) g_{A}$, the growth rate of total factor productivity.

The second GBGP condition requires that factor inputs in the R\&D sector do not change over time. From our specification of the research technology it follows that on a GBGP the rate of innovation must be constant. The equilibrium value of the innovation rate can then be calculated from the arbitrage condition (22). With (18), (19), (25), and the fact that $\boldsymbol{p} . \boldsymbol{Y}=\bar{y}\left(\boldsymbol{p}, \boldsymbol{L}, g_{A}\right) A^{1-\alpha}$, the arbitrage condition can be written as

$$
c_{R}(\overline{\mathbf{w}}) A(t)^{-\alpha}=\int_{t}^{\infty} \alpha(1-\alpha) \bar{y}\left(\boldsymbol{p}, \boldsymbol{L}, g_{A}\right) A(\tau)^{-\alpha} e^{-\int_{t}^{\tau} r(s) d s} d \tau .
$$

In order to solve for the integal in this arbitrage condition, note that along a GBGP the values of $p, \boldsymbol{L}$ and $g_{A}$ are constant, implying that $\bar{y}$ cannot change over time. Furthermore, for a constant rate of innovation we can solve the differential equation (21):

$$
A(\tau)=A(t) e^{g_{A}(\tau-t)} .
$$

Hence, the arbitrage condition simplifies to

$$
c_{R}(\overline{\mathbf{w}})=\alpha(1-\alpha) \bar{y}\left(\boldsymbol{p}, \boldsymbol{L}, g_{A}\right) \int_{t}^{\infty} e^{-\alpha g_{A}(\tau-t)} e^{-\int_{t}^{\tau} r(s) d s} d \tau .
$$

For (33) to hold for any $t$, the interest rate has to be constant. Solving the integral for a constant value of the interest rate gives

$$
\alpha g_{A}+r=\frac{\alpha(1-\alpha)}{c_{R}(\overline{\mathbf{w}})} \bar{y}\left(\boldsymbol{p}, \boldsymbol{L}, g_{A}\right)
$$

We now substitute for $r$ from (8) and arrive at the following relation between the rate of innovation and the growth rate of consumption

$$
\alpha g_{A}+\theta g_{C}+\rho=\frac{\alpha(1-\alpha)}{c_{R}(\overline{\mathbf{w}})} \bar{y}\left(\boldsymbol{p}, \boldsymbol{L}, g_{A}\right) \text {. }
$$

Due to our assumptions on consumer preferences, we have $g_{C}=g_{E}$. Furthermore, clearing of the markets for final goods ensures that $E=\bar{y}\left(\boldsymbol{p}, \boldsymbol{L}, g_{A}\right) A^{1-\alpha}$. The 
latter implies that the growth rate of consumption and the rate of innovation are related by

$$
g_{C}=(1-\alpha) g_{A} .
$$

From (34) and (35) we can then solve for $g_{A}$ as

$$
g_{A}=\left[\alpha(1-\alpha) \frac{\bar{y}\left(\boldsymbol{p}, \boldsymbol{L}, g_{A}\right)}{c_{R}(\overline{\mathbf{w}})}-\rho\right][\alpha+\theta(1-\alpha)]^{-1} .
$$

Eq. (36) implicitly determines the equilibrium rate of innovation as a function of final-goods' prices. For a given price vector $\boldsymbol{p}$, the solution of (36) is unique due to the properties of the $\bar{y}$ function. As shown in appendix $\mathrm{A}$, the equilibrium rate of innovation depends positively on factor endowments $L_{l}(l=1, \ldots, M)$, and negatively on the rate of time preference $\rho$ and the elasticity of substitution $\theta$. This is exactly what we expect from the analysis of aggregated $R \& D$-driven growth models.

\subsection{Restrictions on preferences and production struc- ture}

Our analysis in the previous subsection implicitly assumed that markets for final goods and factor markets clear simultaneously at constant prices $\boldsymbol{p}$. In general, this is not guaranteed without restrictions on consumer preferences and production technologies. We will now work out conditions which are necessary for the existence of a GBGP with simultaneous clearing of all markets at each instant.

We start from the static equilibrium allocation with full diversification. Because along the GBGP relative factor prices are constant, relative factor inputs do not change over time. As shown in appendix B, the production function for final good $i$ can then be written as

$$
Y_{i}=k_{i} A^{1-\alpha} L_{1 i}
$$

where $\left.k_{i} \equiv F_{i}\left(1, \ldots, L_{M i} / L_{1 i}\right)\right]^{1-\alpha}\left(A x_{i} / L_{1 i}\right)^{\alpha}$ is a subsector-specific constant. The production of final good $i$ then grows according to

$$
\frac{\dot{Y}_{i}}{Y_{i}}=(1-\alpha) g_{A}+\frac{\dot{L}_{1 i}}{L_{1 i}}
$$


For goods markets to be in equilibrium along the GBGP, demand and production must grow at identical rates for each final $\operatorname{good} i$ : $\dot{C}_{i} / C_{i}=\dot{Y}_{i} / Y_{i}$. Using (6), (35) and (38), this condition can be written as

$$
\frac{\dot{L}_{1 i}}{L_{1 i}}=\frac{\bar{C}_{i}}{C_{i}}(1-\alpha) g_{A}
$$

(38) implies that changes in factor employments along the GBGP are necessary for structural adjustment to occur. If $\hat{L}_{1 i}=0$ for all $i=1, \ldots, N$, relative outputs of final goods do not change over time. Then (39) says that market clearing for final goods is only possible if $\bar{C}_{i}=0$ for all $i=1, \ldots, N$, i.e. if preferences are homothetic.

Finally, we have a general equilibrium if the growth of factor inputs determined by (39) is compatible with clearing of the factor markets for given factor-input coefficients. We now have to distinguish three cases: (i) the even case, where $N=M$, (ii) the uneven case $N>M$, where $\bar{C}_{i}=0$ for at least $N-M$ final goods, and (iii) the uneven case $N>M$, where $\bar{C}_{i}=0$ for less than $M$ final goods. As we will show in the following, structural adjustment along a GBGP is only possible in case (iii). In cases (i) and (ii), existence of a GBGP requires preferences to be homothetic and thus the cause for structural adjustment-differences in income elasticities of demand-becomes obsolete. Structural adjustment along the GBGP is only possible if income elasticities of demand differ from unity for a sufficient number of goods.

Consider case (i) first. As argued above, the input coefficients $b_{l i}$ are constant along a GBGP since relative factor prices do not change. Furthermore, the amount of ressources allocated to $\mathrm{R} \& \mathrm{D}, \boldsymbol{L}_{R}$, is also required to be constant over time. It follows that the equilibrium conditions for factor markets can be written as

$$
\boldsymbol{B} \overline{\mathbf{Y}}=\boldsymbol{L}-\boldsymbol{L}_{R}
$$

where $\boldsymbol{L}-\boldsymbol{L}_{R}$ is the vector of factor endowments employed in production of intermediates and final goods. Provided that the $N$ final goods are produced by different technologies, the matrix $\boldsymbol{B}$ has full rank, and the productivity-adjusted outputs of final goods $\bar{Y}_{i}$ are fully determined by the conditions for factor-market clearing. Consequently, for a given price vector $\boldsymbol{p}$, the productivity-adjusted levels of final-goods outputs cannot change over time. It follows that $\dot{L}_{1 i} / L_{1 i}=0$ 
for all $i=1, \ldots, N$, and all outputs $Y_{i}$ grow at rate

$$
\frac{\dot{Y}_{i}}{Y_{i}}=(1-\alpha) g_{A}
$$

Obviously, in the even case $N=M$, markets for final goods and factor markets clear simultaneously at constant final-goods' prices iff $\bar{C}_{i}=0$ for all $i$ (cf. (6)), i.e. if preferences are homothetic. The conditions for generalized balanced growth do not allow for structural adjustment. However, if preferences are assumed to be homothetic, the GBGP is identical to the BGP in a sectoral disaggregated model. The model collapses to a generalization of the two-sector growth model of a small open economy developed by Grossman \& Helpman (1991: ch. 6).

A similar argument applies in case (ii). According to the conditions for clearing of the final-goods markets (39), $\bar{C}_{i}=0$ for $i=M+1, \ldots, N$ implies that factor inputs in the respective subsectors do not change along the GBGP. Hence, we can subtract the vector of factor inputs in these subsectors from the endowment vector. Clearing of factor markets then requires

$$
\boldsymbol{B}_{1} \overline{\mathbf{Y}}_{1}=\boldsymbol{L}-\boldsymbol{L}_{R}-\boldsymbol{B}_{2} \overline{\mathbf{Y}}_{2}
$$

where $\boldsymbol{B}_{1}$ is the matrix of input coefficients $b_{l i}(l, i=1, \ldots, M), \boldsymbol{B}_{2}$ is the matrix of input coefficients $b_{l i}(l=1, \ldots, M, i=M+1, \ldots, N), \overline{\mathbf{Y}}_{1}$ is the vector of productivity-adjusted outputs $\bar{Y}_{i}(i=1 \ldots, M)$, and $\overline{\mathbf{Y}}_{2}$ is the vector of productivity-adjusted outputs $\bar{Y}_{i}(i=M+1 \ldots, N)$. Provided that the technologies used in the final-goods subsectors differ, the matrix $\boldsymbol{B}_{1}$ has full rank and the equilibrium conditions for factor markets determine the vector of productivityadjusted outputs. Relative outputs are then again determined by clearing of the factor markets. Again, a GBGP then exists iff $\bar{C}_{i}=0$ for all consumer goods $i=1, \ldots, N$.

Structural adjustment along the GBGP is only possible if $\bar{C}_{i}=0$ for less than $N-M$ subsectors. The problem now is to elaborate on conditions ensuring that the reallocation of factors between subsectors producing final goods required by the growth of outputs is consistent with factor-market clearing. Since input coefficients are fixed and factor markets are in equilibrium initially, we can concentrate on equilibrium on the market for $L_{1}$. The market-clearing condition for this factor requires

$$
\sum_{i=1}^{N} L_{1 i}=L_{1}-L_{1 R}
$$


Differentiating this condition with respect to time and making use of (39) gives

$$
\sum_{i=1}^{N} \frac{\bar{C}_{i}}{C_{i}} L_{1 i}=0 .
$$

By using the market-clearing conditions $C_{i}=Y_{i}$ and (37), we finally arrive at

$$
\sum_{i=1}^{N} \frac{\bar{C}_{i}}{k_{i}}=0 .
$$

This condition is fulfilled under the assumptions we made with respect to consumer preferences, namely $\sum p_{i} \bar{C}_{i}=0$. This can be seen by substituting for $k_{i}$ in (40) as follows. From the condition of equal factor prices across subsectors it follows that marginal value products equalize. With respect to $L_{1}$, we can write this condition (by making use of (37)) as

$$
p_{i} k_{i}=w_{1}, \quad i=1, \ldots, N .
$$

After substituting in (40) for $k_{i}$, the condition for factor-market clearing reduces to

$$
\sum p_{i} \bar{C}_{i}=0 .
$$

This is exactly the same condition that ensures the growth rates of expenditure and of the consumption aggregator to be the same. ${ }^{4}$ In the end, condition (41) ensures factor inputs to adjust such that factor makets clear at each instant.

A special example may be helpful to clarify how the reallocation of factors works. Suppose there are only $M=2$ factors. In this case we can analyze factormarket clearing with the help of a diagram in $\left(L_{1}, L_{2}\right)$ space. In figure 1 , the vector $O \mathcal{L}$ represents the economy's factor endowments. Additionally, we assume that there are $N=3$ final goods. The rays $O \mathcal{A}_{i}(i=1,2,3)$ represent the factor proportions corresponding to a given relative factor price $w_{1} / w_{2}$. We label finalgood subsectors such that subsector 1 uses $L_{1}$ most intensively, and subsector 2

\footnotetext{
${ }^{4}$ Note the difference to the result derived by Kongsamut et al. (1997). These authors also analyze an uneven model (two factors, three goods). Since in their model the accumulation of one factor (capital) is tied to the size of one sector (manufacturing), a GBGP (as defined above) with constant overall growth rate exists only if preferences are homothetic. Hence, these authors take an alternative definition of a GBGP (they take a path with constant rate of interest and implying constant factor intensities) and analyze a path with constant relative price allowing for structural adjustment. However, adding a fourth sector to their model would also allow for structural adjustment and constant overall growth.
} 
uses $L_{2}$ most intensively. Along these rays, the lengths $O \mathcal{B}_{i}=\boldsymbol{b}_{i}\left(\bar{w}_{1}, \bar{w}_{2}\right) \bar{Y}_{i}$ correspond to the factor-input requirements of the equilibrium productivity-adjusted output levels $\bar{Y}_{i}$. Equilibrium on the factor markets then requires that the vector sum of this lengths add up to the endowment vector $O \mathcal{L}$. Obviously, sectoral factor inputs are not uniquely determined by factor market clearing.

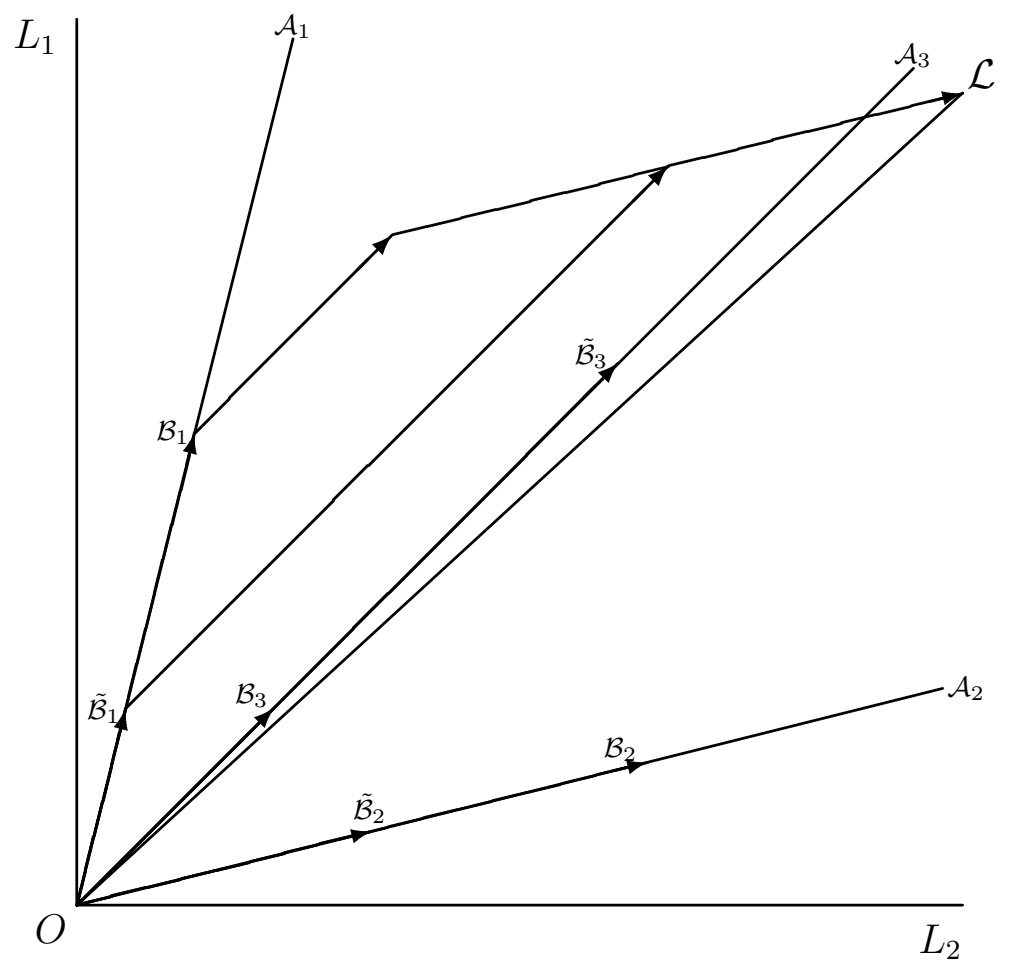

Figure 1: Factor reallocation along the GBGP

Suppose now that in the static equilibrium at $t=0$ the factor inputs in final-goods subsectors are determined by the lengths $O \mathcal{B}_{i}$ which add up to $O \mathcal{L}$ as shown in figure 1 . We know that the GBGP converges to the BGP of the homothetic case with $\bar{C}_{i}=0(i=1,2,3)$ at the same price vector. The homothetic case, however, is characterized by an allocation of factors that does not change over time. Suppose that the factor inputs in the homothetic case are determined by the lengths $O \tilde{\mathcal{B}}_{i}$, which also add up to $O \mathcal{L}$. Starting at $t=0$, the economy starts with the factor allocation characterized by $O \mathcal{B}_{i}$, and converges towards the allocation determined by $O \tilde{\mathcal{B}}_{i}$. Along the GBGP, the lengths $O \mathcal{B}_{i}$ 
monotonically adjust towards their long-run values $O \tilde{\mathcal{B}}_{i}$ with all markets clearing simultaneously at each instant. In the special case illustrated in figure 1, factors are reallocated towards sector 3 as the economy develops along its GBGP. As a result, the productivity-adjusted output of good 3 rises over time. Since we assumed final product 3 to be neither the most $L_{1}$-intensive nor the most $L_{2}$-intensive good, clearing of factor markets requires factor inputs - and hence productivity-adjusted output levels - of subsectors 1 and 2 to decline along the GBGP. This does not imply that production of these goods falls. Due to the increase in total factor productivity by the rise in the number of intermediate goods production of all sectors increases. In our special example, the output of good 3 rises relative to the outputs of the other final goods, and the output of good 2 rises relative to good $1 .^{5}$ Since our choice of factor intensities and our selection of the long-run and the short-run allocation is completely arbitrary, any adjustment driven by preferences fulfilling (41) is compatible with clearing of factor markets.

\section{The model in aggregates}

This section shows that the aggregated version of our model collapses to a special version of the Romer (1990) model. With respect to the reference one-sector model, we depart from Romers original specification in the following way. First, we follow Barro \& Sala-i-Martin (1995: ch. 6) and assume (i) that labor is the only factor of production, and (ii) that intermediate goods are not transformed into physical capital but fully used up in the production of final goods. ${ }^{6}$ Second, we allow for technological differences in the final-goods sector and the intermediate-goods sector by assuming that intermediates are produced using only labor. The latter implies a linear transformation curve between the outputs of intermediates and R\&D. It shows up that our model formulated in aggregates

\footnotetext{
${ }^{5}$ The latter follows from the Rybczynski theorem. With relative factor inputs in subsector 3 exceeding the relative factor endowment (i.e. $L_{13} / L_{23}>L_{1} / L_{2}$ ), the reallocation of factors towards subsector 3 acts like a decline in relative factor endowment that is left for subsectors 1 and 2. According to the Rybczynski theorem, the output of the $L_{2}$ intensive good must then rise relative to the output of the other good.

${ }^{6}$ The second assumption ensures that any form of transitional dynamics is absent in the model.
} 
is formally identical to this modified version of the Romer model. As a result, the analysis of the bahavior of aggregates follows from the appropriately modified version of the Romer model.

In order to prove this assertion, we explicitly carry out the aggregation procedure in our model. Aggregation on the demand side is straightforward. However, we cannot use the already introduced consumption-aggregator function $C$ as a measure of aggregate consumption, because $C$ does not appear in the representative household's budget constraint. Therefore, we apply the Hicksian composite-commodity theorem and define aggregate consumption expenditure as the appropriate consumption index. Given our assumptions on the values of $\bar{C}_{i}$, it is completely irrelevant for the behavior of aggregates whether we define the household's utility function in the aggregated model as a function of $C$ or of $E$. Of course, we can find a price index $P$ for aggregate consumption by some function $\psi(\boldsymbol{p})$. In the following, we take aggregate consumption as numeraire, i.e. we assume $P=1$.

On the production side, we have to aggregate over factor inputs and over factor prices. As shown in appendix B, from the homogeneity properties of the technologies and the fact that relative factor prices do not change along the GBGP it follows that

$$
F_{i}\left(L_{1 i}, \ldots, L_{M i}\right)=b_{i} L_{1 i} .
$$

With respect to the unit-cost functions, this implies

$$
c_{i}\left(w_{1}, \ldots, w_{M}\right)=w_{1} c_{i}\left(1, \ldots, w_{M} / w_{1}\right) .
$$

For constant relative factor prices, $c\left(1, \ldots, w_{M} / w_{1}\right)$ is also a constant value. Due to the linearity of production in $L_{1 i}$, we arrive at

$$
c_{i}\left(w_{1}, \ldots, w_{M}\right)=w_{1} / b_{i} .
$$

The same argument applies for the technologies in the intermediate-goods sector and the research sector. Hence,

$$
\begin{gathered}
c_{x}\left(w_{1}, \ldots, w_{M}\right)=w_{1} / b_{x} \\
c_{R}\left(w_{1}, \ldots, w_{M}\right)=w_{1} / b_{R} .
\end{gathered}
$$

The variables $b_{i} L_{1 i}$ and $w_{1} / b_{i}(i=1, \ldots, N, x, R)$ are a measure of aggregate factor input and aggregate factor payments, respectively. Because of the differences in production technologies the values of $b$ are (sub-) sector specific. In order 
to ensure compatibility to the appropriately modified Romer model, we define $w \equiv w_{1} / b_{x}$ as the wage rate (the price index for aggregate factor inputs), where $b_{x} \equiv\left[c_{x}\left(1, \ldots, w_{M} / w_{1}\right)\right]^{-1}$. As a result, the aggregate factor $b_{x} L_{1}$ is regarded as the economy's labor endowment. Furthermore, the labor-input coefficients of the technologies are given by 1 in the intermediate-goods sector, $b_{x} / b_{R}$ in the R\&D sector, and $b_{x} / b_{i}$ in the $i$ th final-goods sector.

We can then write the unit-cost functions as

$$
\begin{aligned}
c_{x}\left(w_{1}, \ldots, w_{M}\right) & =w \equiv c_{x}(w) \\
c_{R}\left(w_{1}, \ldots, w_{M}\right) & =\eta w \equiv c_{R}(w) \\
c_{i}\left(w_{1}, \ldots, w_{M}, p_{D}\right) & =\kappa_{i} w^{1-\alpha} p_{D}^{\alpha} \equiv c_{i}\left(w, p_{D}\right), \quad i=1, \ldots, N,
\end{aligned}
$$

where $\eta=b_{x} / b_{R}>0$ and $\kappa_{i}>0$ are indexes measuring differences in labor productivity across the various production activities.

We now derive an explicit formula for the rate of innovation in aggregated terms. Denoting the productivity-adjusted real wage by $\bar{w} \equiv w A^{\alpha-1}$, the productivity-adjusted net GDP function can then be written as ${ }^{7}$

$$
\begin{gathered}
\bar{y}\left(L, g_{A}\right)=\gamma\left[\bar{w} L-g_{A} c_{R}(\bar{w})\right] \\
\Longleftrightarrow \\
\bar{y}\left(L, g_{A}\right)=\gamma\left[\bar{w}\left(L-L_{R}\right)\right] .
\end{gathered}
$$

From that we derive

$$
\frac{\bar{y}\left(L, g_{A}\right)}{c_{R}(\bar{w})}=\gamma\left(\frac{L}{\eta}-g_{A}\right) .
$$

Substituting in (36), we arrive at

$$
g_{A}=\left[\frac{\alpha(1-\alpha)}{1-\alpha(1-\alpha)} \frac{L}{\eta}-\rho\right]\left[\alpha\left(1+\frac{1-\alpha}{1-\alpha(1-\alpha)}\right)+\theta(1-\alpha)\right]^{-1}
$$

Formally, this is exactly the same result which can be derived from the version of the Romer model modified as discussed above. ${ }^{8}$ Analogously, we can derive the results for the growth rates of aggregate consumption and the real wage,

\footnotetext{
${ }^{7}$ We can omitt the price index $P$ as argument of the productivity-adjusted net GDP function since $P$ has been normalized to unity.

${ }^{8}$ The result differs slightly from the result derived by Barro \& Sala-i-Martin (1995, ch. 6) since we do not assume identical technologies for producing intermediates and final goods.
} 
and arrive at the same results as in the (appropriately modified) Romer model. Hence, all results derived from the non-disaggregated Romer model carry over to the present model formulated in aggregates. Consequently, the Romer model describes correctly the behavior of aggregates in our disaggregated model. If we are only interested in the behavior of aggregate variables - such as the innovation rate, consumption expenditure, aggregate factor incomes - there is no need to analyse the GBGP of the disaggregated model. We can take the results directly from the BGP analysis of the non-disaggregated model.

\section{Conclusion}

Critics of growth theory stress the massive change in sectoral composition as a challenge for theories concentrating on balanced-growth paths (BGP). Our analysis has shown that the obvious inconsistence of these models and structural adjustment does not necessarily render BGP analyses useless. By disaggregating the final-goods sector of a standard research-driven growth model and generalizing the concept of a balanced growth path (GBGP), we could explain that balanced-growth behavior of aggregate variables is indeed consistent with a massive change in sectoral composition of the economy. In this sense, structural adjustment is only a byproduct of economic growth that has no feedback on the growth process itself. This stands in contrast to Schumpetarian growth models (cf. Aghion \& Howitt 1998), where a special form of structural change within the intermediate -goods sector - the permanent change in the industry leadership by the process of "creative destruction" - drives economic growth. However, we can easily substitute for the product-variety explanation of growth by the Schumpetarian specification where innovations take the form of quality upgrading of intermediate goods thus covering this additional aspect of structural change.

The decisive prerequisite for the explanation of structural change along a GBGP in the present model is that the production-sector equilibrium is not uniquely determined for a given vector of goods' prices and given factor endowments. With different production technologies, such an indeterminacy arises when the number of final goods used for consumption purposes exceeds the number of factors. Indeterminacy of the production-sector equilibrium, however, is not a necessary condition for structural adjustment along a GBGP. Alternatively, we 
can give up our assumption of exogenously given factor supplies and search for a suitable modelling of factor accumulation in a model with an equal number of factors and final goods. ${ }^{9}$ In such a framework, outputs are uniquely determined by factor-market clearing at each point in time. However, relative outputs of final goods will change if the accumulation process alters the economy's relative factor endowments over time. Of course, we can always specify a process of factor accumulation ad hoc generating a path of final-goods' outputs that is consistent with consumers' demand derived from nonhomothetic preferences. The challenge of this modification is to provide a plausible microeconomic foundation, or other plausible justification, for the process of factor accumulation that is compatible with the required growth of final-goods outputs. This is the program of ongoing research.

\section{References}

Aghion, P., and Howitt, P. (1992) "A model of growth through creative destruction", Econometrica 60, 323-351.

Aghion, P., and Howitt, P. (1998) Endogenous growth theory, MIT Press: Cambridge/Mass.

Barro, R.J., and Sala-i-Martin, X. (1995) Economic growth, McGraw-Hill: New York.

Baumol, W.J., Blackman, A.B., and Wolf, E.N. (1989) Productivity and american leadership in the world, MIT Press: Cambridge/Mass.

Chenery, H.B. (1960) "Patterns of industrial growth", American Economic Review 50, 624-653.

Clark, C. (1957) The conditions of economic progress, 3rd. ed., McGraw-Hill: London.

Echevarria, C. (1997) "Changes in sectoral composition associated with economic growth", International Economic Review 38, 431-452.

Grossman, G.M., and Helpman, E. (1991) Innovation and growth in the global economy, MIT Press: Cambridge/Mass.

\footnotetext{
${ }^{9}$ Note that with growing factor endowments we have to modify our specification of the R\&D technology in order to avoid explosive growth rates. This can be done along the lines suggested by Jones (1995).
} 
Jones, C.I. (1995) "R\&D-based models of economic growth," Journal of Political Economy 103, 759-784.

Jones, C.I. (1997) "The upcoming slowdown in U.S. economic growth," NBER Working Paper 6284.

Kuznets, S. (1971) Economic growth of nations: total output and production structure, The Belknap Press of Harvard University Press: Cambridge/Mass.

Kongsamut, P., Rebelo, S., and Xie, D. (1997) "Beyond balanced growth," NBER Working Paper 6159.

Rivera-Batiz, L.A. and Romer, P.M. (1991) "Economic integration and economic growth," Quarterly Journal of Economics 106, 531-555.

Romer, P.M. (1990) "Endogenous technological change," Journal of Political Economics 98, S71-S102. 


\section{Appendix}

\section{A Deriving the properties of the equilibrium rate of innovation}

Calculating the comparative-static effects of changes in factor endowments, in the rate of time preference, and in the elasticity of substitution from (36) gives

$$
\begin{aligned}
\frac{d g_{A}}{d L_{l}} & =\frac{\alpha(1-\alpha)}{c_{R}(\overline{\mathbf{w}})} \frac{\partial \bar{y}(.)}{\partial L_{l}}[\alpha+\theta(1-\alpha)]^{-1} \psi, \quad l=1, \ldots, M \\
\frac{d g_{A}}{d \rho} & =-[\alpha+\theta(1-\alpha)]^{-1} \psi \\
\frac{d g_{A}}{d \theta} & =-\alpha(1-\alpha)^{2} \frac{\bar{y}(.)}{c_{R}(\overline{\mathbf{w}})}[\alpha+\theta(1-\alpha)]^{-2} \psi,
\end{aligned}
$$

where

$$
\psi \equiv\left\{1-\frac{\alpha(1-\alpha)}{c_{R}(\overline{\mathbf{w}})} \frac{\partial \bar{y}(.)}{\partial g_{A}}[\alpha+\theta(1-\alpha)]^{-1}\right\}^{-1} .
$$

The derivative properties of the net GDP function ensure that $d g_{A} / d L_{l}>0$, $d g_{A} / d \rho<0$, and $d g_{A} / d \theta<0$.

\section{B The production function at constant relative prices}

Due to the linear-homogeneity of the functions $F_{i}$ in factor inputs, production of $Y_{i}$ can be written as:

$$
Y_{i}=\left[F_{i}\left(1, \ldots, L_{M i} / L_{1 i}\right)\right]^{1-\alpha} L_{1 i}^{1-\alpha} A x_{i}^{\alpha} .
$$

For constant relative factor prices, the term $F_{i}\left(1, \ldots, L_{M i} / L_{1 i}\right)$ is a sector-specific constant. Hence, the growth rate of final-goods output $i$ along the GBGP is given by

$$
\frac{\dot{Y}_{i}}{Y_{i}}=(1-\alpha) \frac{\dot{L}_{1 i}}{L_{1 i}}+g_{A}+\alpha \frac{\dot{x}_{i}}{x_{i}}
$$

The growth rate of $x_{i}$ along the GBGP can be calculated from (15):

$$
\frac{\dot{x}_{i}}{x_{i}}=\frac{\dot{Y}_{i}}{Y_{i}}+\frac{\alpha}{1-\alpha} \frac{\dot{p}_{D}}{p_{D}}-\frac{1}{1-\alpha} \frac{\dot{p}_{x}}{p_{x}} .
$$


With

$$
p_{D}=\frac{c_{x}(\bar{w})}{\alpha} A^{-(1-\alpha)^{2} / \alpha}
$$

from (17) and (24), and

$$
p_{x}=\frac{c_{x}(\bar{w})}{\alpha} A^{1-\alpha}
$$

from (16) and (24), we arrive at

$$
\frac{\dot{x}_{i}}{x_{i}}=\frac{\dot{Y}_{i}}{Y_{i}}-(2-\alpha) g_{A}
$$

Hence,

$$
\frac{\dot{Y}_{i}}{Y_{i}}=\frac{\dot{L}_{1 i}}{L_{1 i}}+(1-\alpha) g_{A}
$$

Substituting back, we get

$$
\frac{\dot{x}_{i}}{x_{i}}=-g_{A}+\frac{\dot{L}_{1 i}}{L_{1 i}}
$$

which implies that $A x_{i} / L_{1 i}$ is constant. Rewriting (B.1) as

$$
Y_{i}=\left[F_{i}\left(1, \ldots, L_{M i} / L_{1 i}\right)\right]^{1-\alpha} L_{1 i} A^{1-\alpha}\left(\frac{A x_{i}}{L_{1 i}}\right)^{\alpha},
$$

and using the fact that along the GBGP $A x_{i} / L_{1 i}$ is constant, we finally arrive at

$$
Y_{i}=k_{i} A^{1-\alpha} L_{1 i}
$$

where $k_{i} \equiv\left[F_{i}\left(1, \ldots, L_{M i} / L_{1 i}\right)\right]^{1-\alpha}\left(A x_{i} / L_{1 i}\right)^{\alpha}$ is a subsector-specific constant. 